\title{
Analysis of regional left ventricular wall motion during diastole in mitral stenosis
}

\author{
W K K HUI^, P K LEE†, J S F CHOW†, D G GIBSON \\ From Brompton Hospital, London
}

SUMMARY Left ventricular angiograms of 16 patients with mitral stenosis and 18 normal subjects were digitised frame by frame to study regional diastolic wall motion. Left ventricular cavity volume and its rate of change were derived, and plots constructed to show regional wall motion throughout the cardiac cycle. Patients with mitral stenosis had a significantly reduced peak filling rate of $380 \pm 170 \mathrm{ml} / \mathrm{s}$, in comparison with normal $(690 \pm 260 \mathrm{ml} / \mathrm{s})$. A slow rate of filling, however, was not associated with slow and uniform outward movement of the endocardium of the left ventricle. In patients with mitral stenosis, peak outward velocities were normal at the apex and along the inferior wall, and only slightly reduced anteriorly $(9 \pm 3 \mathrm{~cm} / \mathrm{s}$, normal $11 \pm 4 \mathrm{~cm} / \mathrm{s})$. Instead, striking nonuniformity of wall motion was present, so that the interval over which peak wall motion occurred in the three regions of the cavity was extended to $93 \pm 53 \mathrm{~ms}$ (normal $43 \pm 25 \mathrm{~ms}$ ). The same applied to the termination of rapid outward movement, which occurred over an interval of $110 \pm 44 \mathrm{~ms}$ (normal $43 \pm 21)$. A second manifestation of regional asynchrony was abnormal outward wall movement during isovolumic relaxation, and in nine patients it had reached its peak velocity in at least one region before the mitral valve opened. Finally, the ventricular cavity was seen to oscillate as it filled in six patients, with the endocardium moving inwards in one region after mitral valve opening associated with accentuated outward movement elsewhere. The reduced left ventricular filling rate in mitral stenosis thus manifests itself in small part only as slow outward wall motion. The main disturbance to regional left ventricular function was a reproducible series of disturbances causing striking asynchrony. This dissociation of regional wall motion from filling and the constant finding of motion of the anterior free wall of the ventricle preceding that elsewhere are similar to the disturbances seen in patients with coronary artery disease. Both may represent disorganisation in disease of the highly specialised restoring forces whose action is the basis of coordinate filling in the normal heart.

Abnormal left ventricular filling has long been recognised as the fundamental factor responsible for impaired cardiac function in mitral stenosis. It has variously been assessed by the measurement of a diastolic pressure gradient across the mitral valve and calculation of valve area, 1 by demonstration of an abnormal pattern of left ventricular dimension change, ${ }^{2}$ and by determination of flow velocity across the mitral valve by Doppler techniques. ${ }^{34}$ Left ventricular diastolic filling rate has been shown angiog-

*The work was performed during the tenure of a Croucher Foundation Fellowship.

†Present address: University Medical Unit, Queen Mary Hospital, Hong Kong.

Accepted for publication 26 May 1983 raphically to be decreased in mitral stenosis, ${ }^{5}$ but little has been done to analyse the implications of this reduction in terms of regional wall motion. The aim of the present study was to investigate this aspect in more detail, not only in view of its practical significance as a cause of reduced exercise tolerance but also to take advantage of the presence of the relatively simple but clearly defined modification of left ventricular inflow in patients with mitral valve disease to gain further insight into mechanisms underlying filling in the normal heart.

\section{Subjects and methods}

Sixteen patients with rheumatic mitral stenosis who 
had undergone cardiac catheterisation at the Department of Medicine, University of Hong Kong, as part of their routine preoperative assessment were studied. Their ages ranged from 24 to 70 years. There were eight in sinus rhythm and eight in atrial fibrillation. None had a history of angina pectoris or hypertension. Eighteen patients with chest pain who at cardiac catheterisation were found to have normal haemodynamics and normal coronary arteriograms were taken as normal controls.

Left ventricular angiograms were performed in the 30 degree right anterior oblique projection with the patients supine. A bolus of 20 to $40 \mathrm{ml}$ contrast was injected at a rate of 12 to $15 \mathrm{ml} / \mathrm{s}$ through a retrograde catheter into the left ventricle with cine film exposed at 50 frames a second. Calibration was performed using a grid at midchest level. No patient had significant mitral regurgitation. Only normally conducted beats were selected for analysis. Ectopic and post-ectopic beats were not studied.

\section{ANALYSIS OF ANGIOGRAMS}

Left ventricular angiograms were digitised using a Prime 750 computer and a Summagraphics digitiser. ${ }^{6}$ Successive frames of the beat to be studied were projected on to the digitising table, and the opacified left ventricular cavity outlines were traced with the cursor. Strings of coordinates were generated for approximately 50 to 100 points on each frame, together with a reference point on the border of the cine frame. The

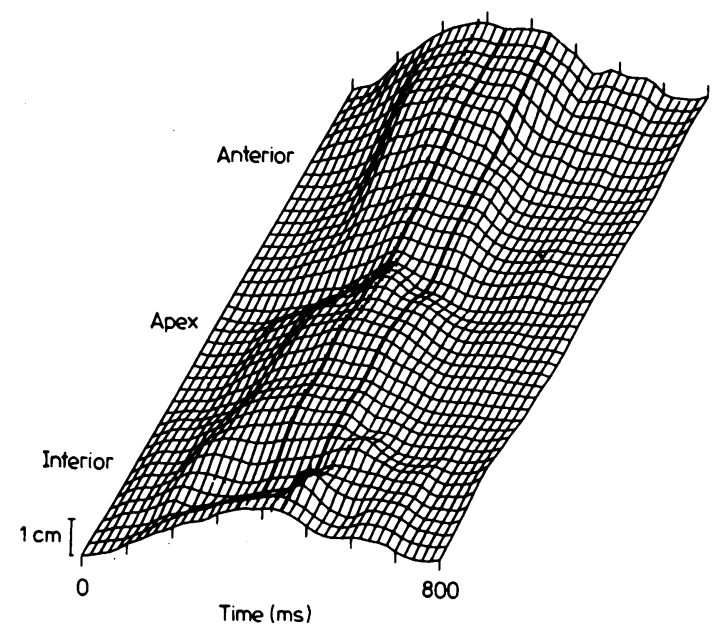

Fig. 1 Isometric display of regional wall movement against time from a normal left ventriculogram. The diagonal lines are isochrones, connecting events occurring simultaneously. The two accentuated isochromes represent the times of mirimum area and mitral valve opering.

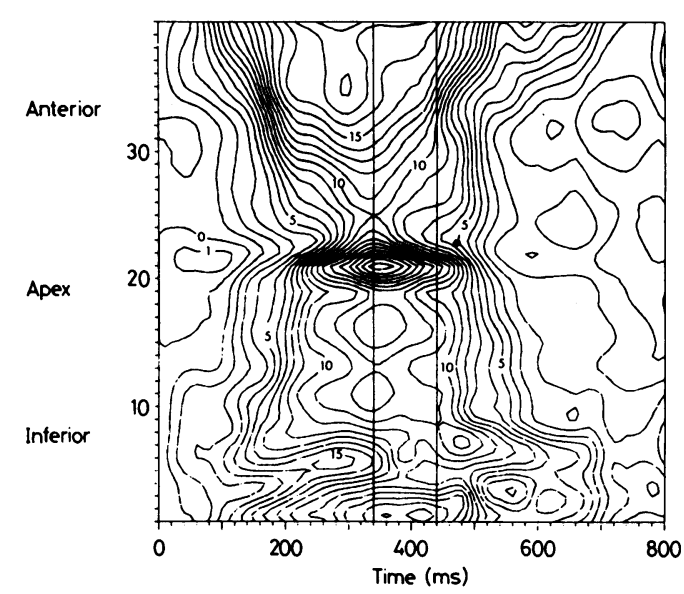

Fig. 2 Contour display of inward wall movement with position around the cavity, and time from the same data as in Fig. 1. Inward wall movement is indicated by contour lines, which represent distances in $\mathrm{mm}$ shown by the figures, from the position of the wall in the frame with maximum cavity area. The vertical lines represent the times of minimum cavity and mitral valve opening.

information was stored on disk and from it the following were derived:

(i) Superimposed plots of cavity outlines during systole and diastole.

(ii) Graphs showing changes of cavity area, derived volume, shape index, ${ }^{6}$ major axis, and the rate of change of volume throughout the cardiac cycle. On these graphs were superimposed the timings of minimum cavity area and mitral valve opening, taken as the time of first appearance of unopacified blood in the left ventricle from the left atrium.

(iii) Plots showing regional movement of the left ventricular endocardium. The rationale and limitations of this method have been described elsewhere. It depends on defining $\mathbf{4 0}$ equally spaced points on the end-diastolic cavity outline starting from the border of the aortic root adjacent to the mitral valve, and proceeding in an anticlockwise direction. From each the nearest point on the end-systolic frame was sought, and along the line thus defined, endocardial position in each cine frame was measured and expressed in $\mathrm{mm}$ from its end-diastolic position. The resulting 40 plots of wall movement against time were displayed either stacked obliquely, as an isometric display with superimposed isochrones to represent the timing of successive cine frames (Fig. 1) or as a contour map (Fig. 2), where each contour represents inward movement of the endocardium by $1 \mathrm{~mm}$ from its end-diastolic position. On both displays, the times of minimum cavity area and mitral valve opening were marked. 

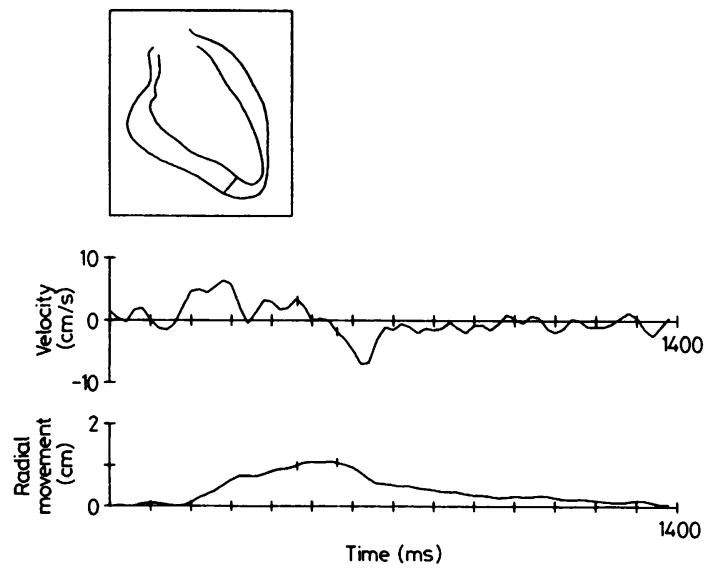

Fig. 3 Plots of local wall movement and velocity against time from the apical region of a patient with mitral stenosis. From below, panels represent change in wall position and rate of change of position with time. The origin of the trace is shown superimposed on end-diastolic and end-systolic outlines in the figure at the top. The times of minimum cavity area and mitral valve opening are indicated by the two vertical markers.

For the purpose of regional wall motion analysis, the left ventricular endocardial outline was divided into three regions: inferior, apical, and anterior, corresponding to segments 1 to 14,15 to 24 , and 25 to 40 , respectively, from the $\mathbf{4 0}$ points on the diastolic frame. The velocity of inward wall movement against time from the midpoint of each region was plotted separately (Fig. 3). The following measurements were made for each of the three regions.

(a) Peak velocity of outward wall movement and its timing after mitral valve opening. If peak velocity occurred before mitral valve opening this time was expressed as a negative value. The time inerval between the earliest and latest occurrence of peak velocity between the three regions, the spread, was also determined.

(b) Velocity of outward wall movement at mitral valve opening.

(c) Rapid outward wall movement was taken as having terminated when its velocity had dropped to less than $3 \mathrm{~cm} / \mathrm{s}$. The time when this occurred for each region and its spread between regions were noted.

(d) Abnormalities of segmental wall movement during the isovolumic relaxation period (time interval between the occurrence of minimum cavity area and mitral valve opening), defined as involvement of four or more consecutive segments in inward movement of more than $2 \mathrm{~mm}$ or outward movement of more than 6 mm during this period. ${ }^{7}$

(e) Inward wall movement of more than $2 \mathrm{~mm}$ after mitral valve opening, that is during the period of ventricular filling. ${ }^{7}$

Comparisons were made between the three different regions of the ventricle and also between normal subjects and patients with mitral stenosis.

\section{STATISTICAL METHODS}

Results were analysed using either standard $t$ tests or Mann-Whitney U tests.

\section{Results}

\section{(A) NORMAL SUBJECTS}

(i) Overall function

The heart rate of the beats digitised was $64 \pm 12 / \mathrm{min}$ (mean $\pm 1 \mathrm{SD})$. Peak diastolic filling rate $(\mathrm{dVol} / \mathrm{dT})$, as derived from volume curves, was $690 \pm 260 \mathrm{ml} / \mathrm{s}$. This occurred $58 \pm 34 \mathrm{~ms}$ After mitral valve opening, that is early in diastole rather than in atrial systole in all patients. End-diastolic volume (EDV) was $165 \pm 55 \mathrm{ml}$ and the peak filling rate divided by end-diastolic volume (dVol/dT per unit EDV) was $4 \cdot 2 \pm 0.65 / \mathrm{s}$. Overall shape index change during the cardiac cycle was $22 \pm 7 \%$, with $8 \pm 3 \%$ occurring in the isovolumic relaxation period.

\section{(ii) Regional function}

The velocities of outward wall motion at the time of mitral valve opening along the anterior, apical, and inferior regions were $7 \pm 5 \mathrm{~cm} / \mathrm{s}, 5 \pm 5 \mathrm{~cm} / \mathrm{s}$, and $5 \pm 4$ $\mathrm{cm} / \mathrm{s}$. Peak values of $11 \pm 4 \mathrm{~cm} / \mathrm{s}, 11 \pm 5 \mathrm{~cm} / \mathrm{s}$, and $13 \pm 4 \mathrm{~cm} / \mathrm{s}$ occurred $39 \pm 33 \mathrm{~ms}, 40 \pm 28 \mathrm{~ms}$, and $43 \pm 27 \mathrm{~ms}$, respectively, after mitral valve opening. With the exception of the inferior region in one patient, peak velocity of outward movement never occurred before mitral valve opening. Rapid outward wall motion ended at $101 \pm 28 \mathrm{~ms}, 94 \pm 27 \mathrm{~ms}$, and $89 \pm 38 \mathrm{~ms}$ in the respective regions after mitral valve opening. There was no statistical difference between the velocities or the timings when the three regions were compared.

The spread in the timing of peak outward velocity between the three regions was $43 \pm 25 \mathrm{~ms}$ and that for the end of rapid outward movement was $43 \pm 21 \mathrm{~ms}$.

Wall motion during the isovolumic relaxation period was within previously published normal limits ${ }^{7}$ in all subjects.

(B) MITRAL STENOSIS

(i) Overall function

In patients with mitral stenosis the heart rate of the beats digitised was $70 \pm 19 / \mathrm{min}$, not significantly different from normal. Peak left ventricular filling rate was greatly reduced to $380 \pm 170 \mathrm{ml} / \mathrm{s}(p<0.001$ with respect to normal). The timing of peak values, however, at $73 \pm 36 \mathrm{~ms}$ after mitral valve opening, was 

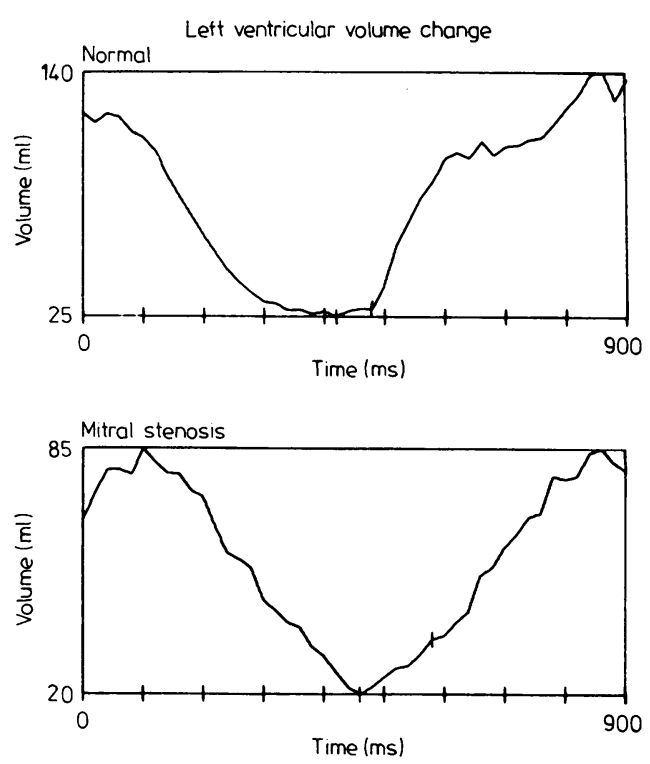

Fig. 4 Plots of left ventricular volume change against time in a normal subject and in a patient with mitral stenosis. The two vertical markers represent the times of minimum cavity area and mitral valve opening. Note that the normal early rapid filling phase after mitral valve opening is absent in mitral stenosis where slow and continuous diastolic filling occurs.

normal (Fig. 4 and 5). Again the peak occurred early in diastole in all patients and not during atrial systole. Though end-diastolic volume was smaller than the normal group at $108 \pm 29 \mathrm{ml}(\mathrm{p}<0.05)$, when peak filling rate was corrected for end-diastolic volume the value of $3.6 \pm 1 \cdot 1 / \mathrm{s}$ was still significantly less than normal $(p<0.05)$. Overall change of shape index during the cardiac cycle was $18 \pm 6 \%$, with $7 \pm 4 \%$ occurring during isovolumic relaxation. These values were not significantly different from normal. There were no significant differences between patients in sinus rhythm and those in atrial fibrillation.

\section{(ii) Regional functions}

There was no significant difference in overall amplitude of movement during the cardiac cycle between the three regions. The velocities of outward wall motion at mitral valve opening were $4 \pm 5 \mathrm{~cm} / \mathrm{s}, 4 \pm 3$ $\mathrm{cm} / \mathrm{s}$, and $2 \pm 6 \mathrm{~cm} / \mathrm{s}$ along the anterior, apical, and inferior regions, respectively, reaching peaks of $9 \pm 3$ $\mathrm{cm} / \mathrm{s}, 10 \pm 3 \mathrm{~cm} / \mathrm{s}$, and $11 \pm 4 \mathrm{~cm} / \mathrm{s}$. There was no statistical difference between values for the three regions. Compared with normal subjects only the outward velocity of the anterior region showed any significant decrease $(p<0.05$ for both velocity at mitral valve opening and peak velocity).

The three regions neither reached peak outward velocity nor ended rapid outward movement at the same time. Peak outward velocity occurred at $-8 \pm 40$ $\mathrm{ms}, 21 \pm 54 \mathrm{~ms}$, and $50 \pm 55 \mathrm{~ms}$ after mitral valve opening in the anterior, apical, and inferior regions, respectively, and the corresponding times for termination of rapid outward movement were $37 \pm 55 \mathrm{~ms}$, $66 \pm 55 \mathrm{~ms}$, and $100 \pm 60 \mathrm{~ms}$. In nine out of 16 patients at least one region reached peak outward velocity before mitral valve opening. Of these, two patients showed this phenomenon in both the anterior and apical regions. The anterior region was involved on seven occasions and the apex on four occasions. In general, the anterior region was usually the first to reach peak outward velocity and end rapid outward movement while the inferior region was usually the last to do so. These differences were statistically significant $(p<0.002$ for peak outward velocity and p $<0.02$ for termination of rapid outward movement). Compared with normal subjects, these two events also occurred earlier in the anterior region in patients with mitral stenosis ( $p<0.002$ for both). The time spread in the occurrence of peak outward velocity was $93 \pm 53$ $\mathrm{ms}$, significantly wider than normal $(\mathrm{p}<0.02)$. The corresponding value for end of rapid outward movement was $110 \pm 44 \mathrm{~ms}$, again much wider than normal $(\mathbf{p}<0.002)$. This much wider spread in timing reflects the notable asynchrony in diastolic events in patients with mitral stenosis.
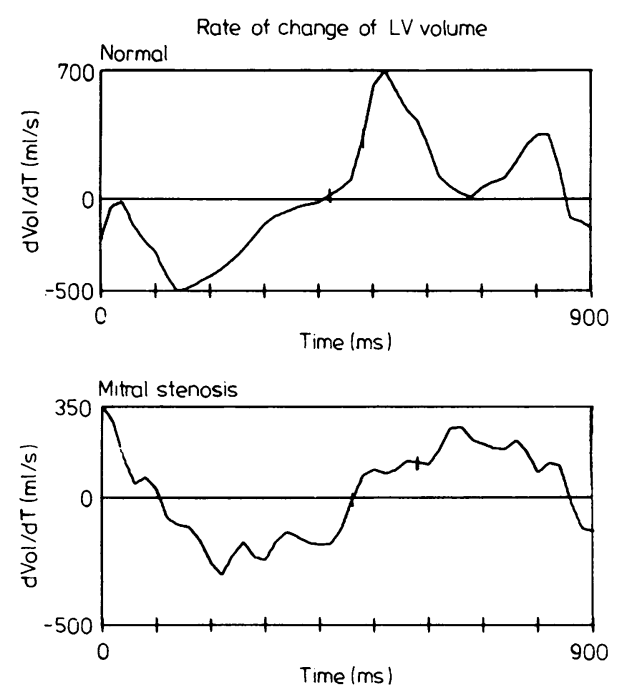

Fig. 5 Plots of rate of change of left ventricular volume against time (dVol/dT) for the same patients as in Fig. 3. The early rapid filling phase in the normal subject is reflected in a much higher peak filling rate than in the patient with mitral stenosis. The times of minimum cavity area and mitral valve opening are indicated by the two vertical markers. 
Abnormal inward wall movement during the isovolumic relaxation period was found in six patients, the anterior region being involved on one occasion, the apical region on four occasions, and the inferior region on one occasion. No patient had involvement of more than one region. Significant outward wall movement in this period occurred in three patients, again not involving more than one region. The anterior region was involved on two occasions and the apical region on one occasion. (iii) Ventricular oscillations

Six patients showed inward movement of more than $2 \mathrm{~mm}$ during filling, involving at least one region in four and two regions in two. The anterior region was involved on four occasions, the apical region on one occasion, and the inferior on three occasions. This inward movement was accompanied by outward movement in another region and gave rise to a diastolic oscillatory movement of the left ventricular wall, illustrated on isometric and contour plots (Fig. 6 and

(a)
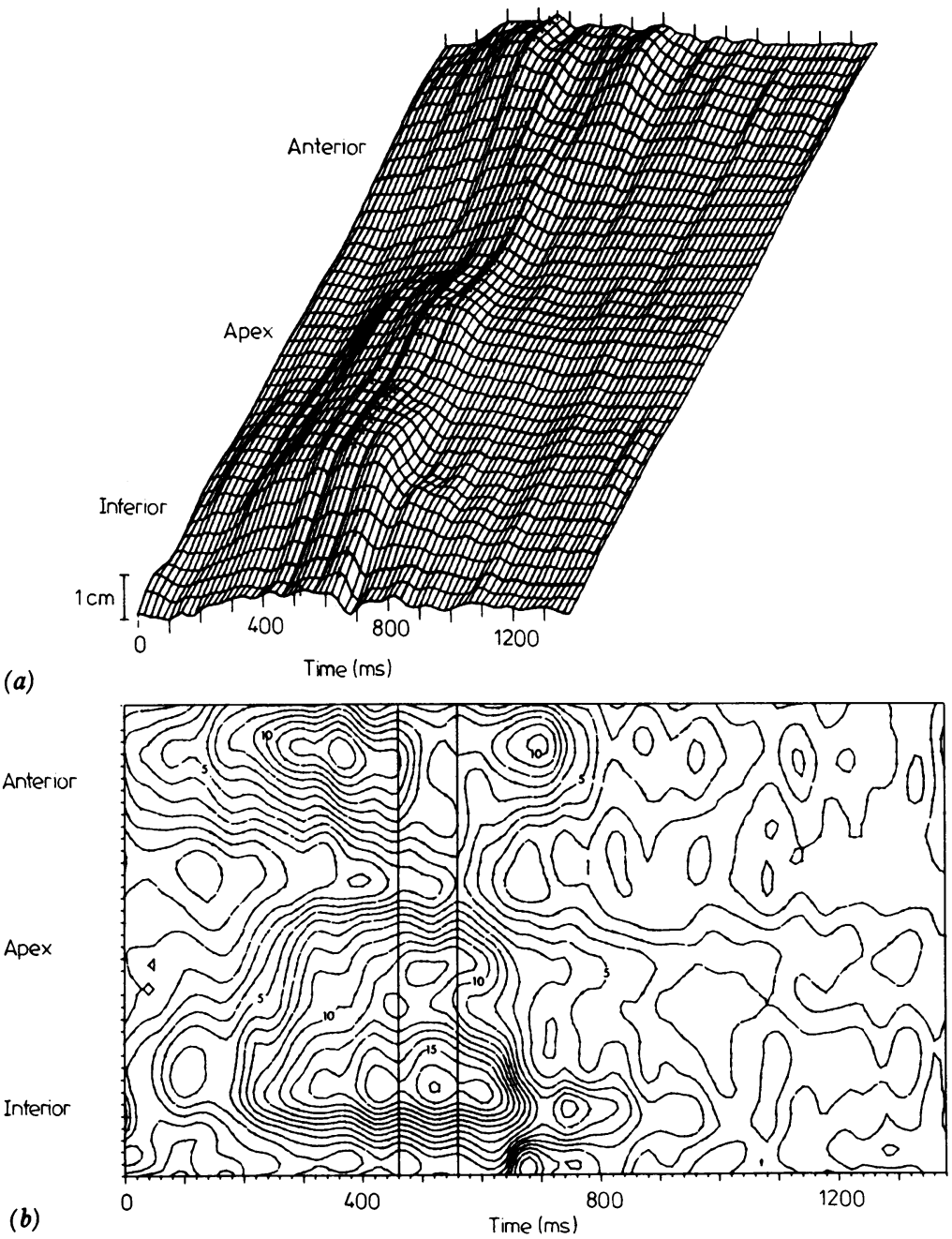

Fig. 6 Isometric (a) and contour (b) display of wall movement against time from a patient with mitral stenosis. Note the abnormal early outward movement starting during isovolumic relaxation period in the anterior region. This is followed by inward movement after mitral valve opening accompanied by compensatory outward movement in the inferior region resulting in an oscillation. 
(a)
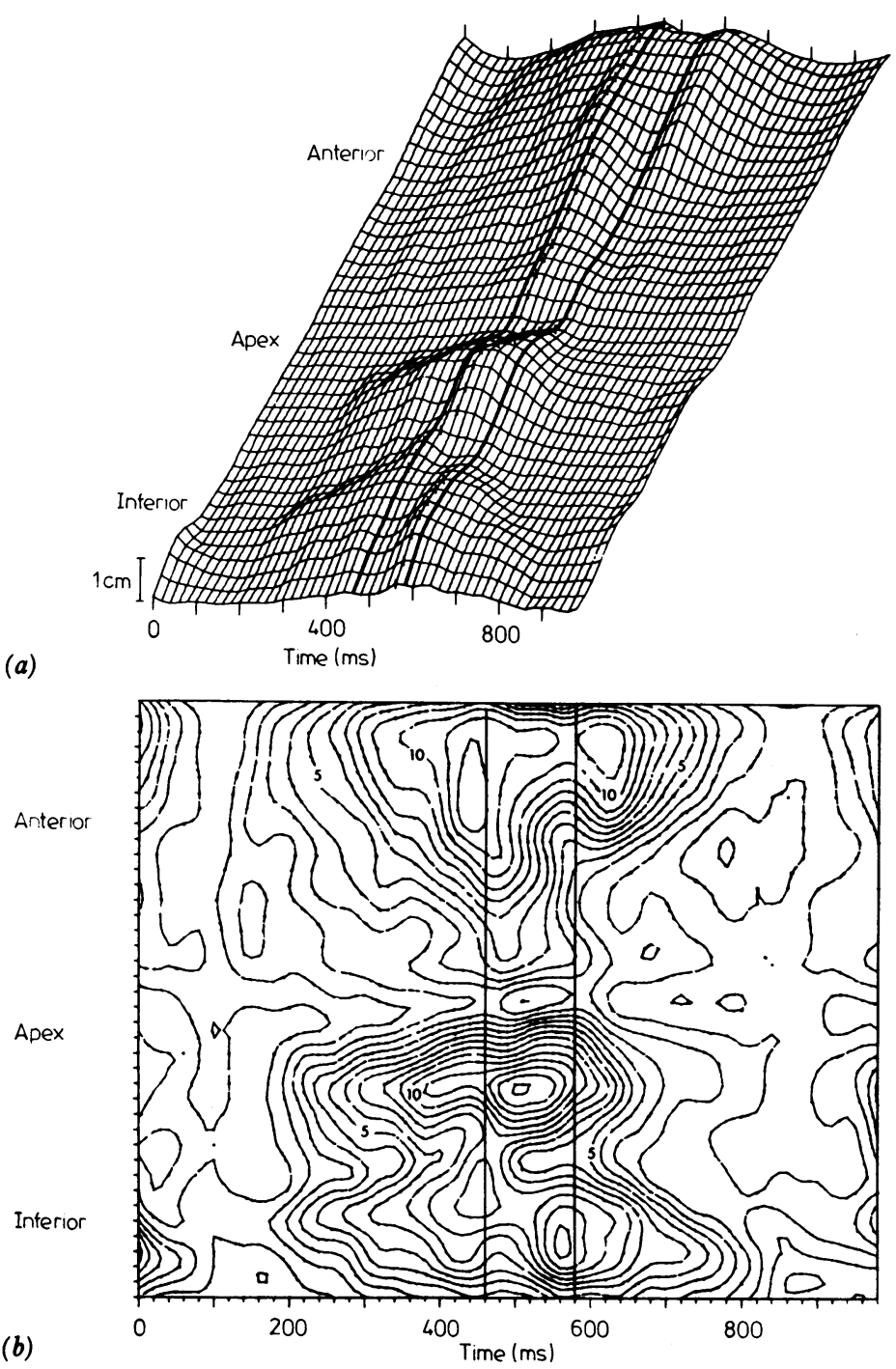

Fig. 7 Isometric ( $a$ ) and contour (b) display of wall movement against time from another patient with mitral stenosis. The anterior wall oscillation is more pronounced, and again accompanied by compensatory inferior motion. 
(a)
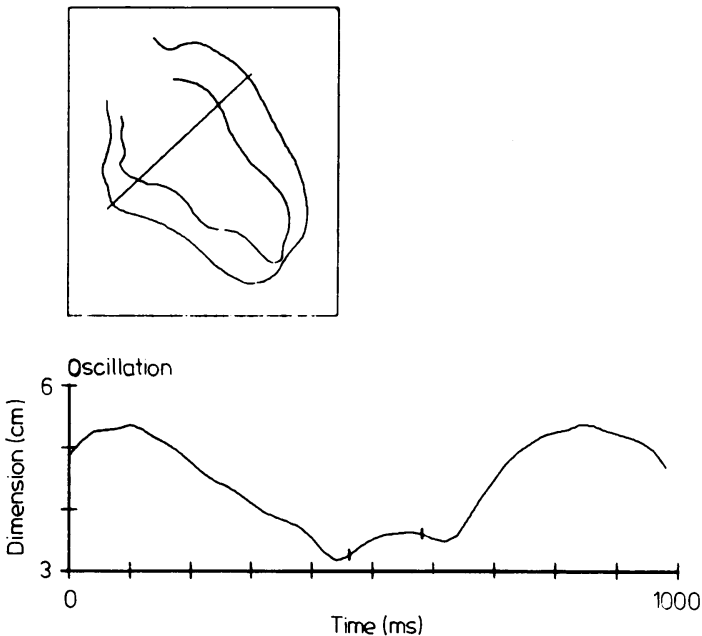

No oscillation
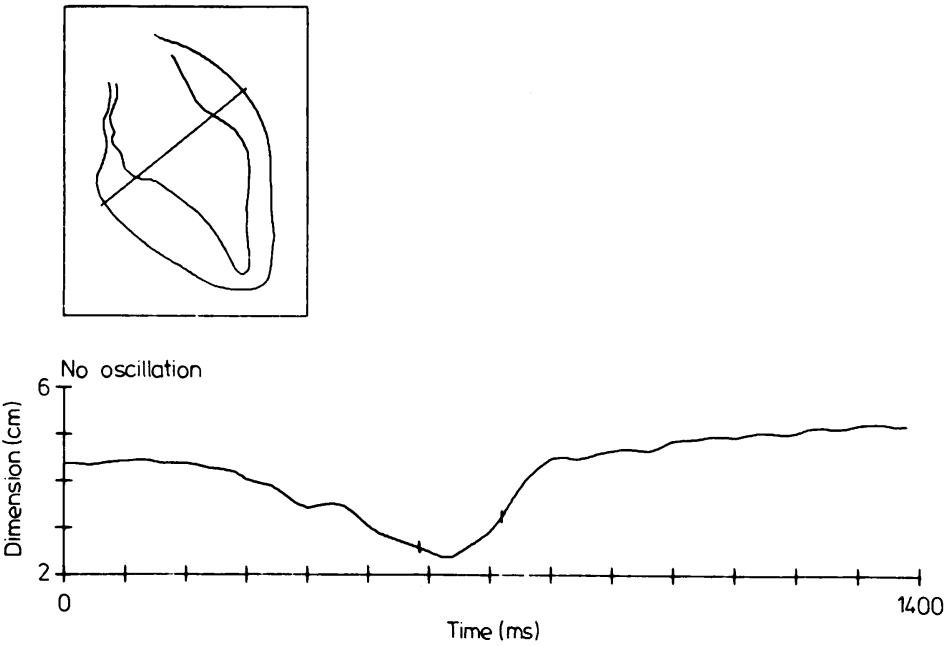

Fig. 8 Plots of left ventricular dimension against time from two patients with mitral stenosis, one with ventricular oscillation (a) and one without (b). Plot (a) is from the patient whose isometric and contour displays are seen in Fig. 7. Note the decrease in ventricular dimension after mitral valve opening coinciding with delayed inward movement in the anterior region. Such a dimension change is not seen in $(b)$. The times of minimum cavity area and mitral valve opening are indicated by the two vertical markers. The origin of the traces is shown superimposed on end-diastolic and end-systolic outlines in the upper panels. 
7). These oscillations were not the result of overall motion of the heart in space, since left ventricular transverse dimension also showed similar behaviour (Fig. 8).

\section{Discussion}

Diastolic filling in the normal left ventricle is a complex but well coordinated and synchronous process. Our results, in line with previous studies, ${ }^{7}$ showed no abnormal regional wall motion during isovolumic relaxation in normal subjects. Early rapid filling was reflected in early rapid outward wall movement, which tended to start before mitral valve opening particularly in the regions along the free wall visualised in the right anterior oblique projection, though peak velocities were always reached during filling. The degree of synchrony of outward wall motion was shown by the small regional differences with respect to the times of peak outward velocity and termination of rapid outward movement.

Patients with mitral stenosis have a simple mechanical obstruction to left ventricular filling. In the present study none had any clinical evidence of coronary artery disease, which in any case is uncommon in Chinese. ${ }^{8}$ If it is assumed that diastolic flow directly determines endocardial position during left ventricular filling it would be anticipated that wall motion during the isovolumic relaxation period would be normal and that following mitral valve opening would be slow but uniform. Our results departed strikingly from these simple predictions. The patients did indeed have obstruction to filling, as was shown by the reduced filling rate, but of the three regions, only the anterior showed any significant decrease in peak outward velocity and this only to $9 \mathrm{~cm} / \mathrm{s}$ against a normal of $11 \mathrm{~cm} / \mathrm{s}$. Instead, pronounced loss of uniformity appeared. During isovolumic relaxation, abnormal wall motion, both inward and outward, could be observed. In more than half of the patients peak rates of outward wall motion were reached before mitral valve opening in at least one region, a feature that was almost never observed in normal subjects. After mitral valve opening, the reduction in the rate of cavity volume increase manifested itself as asynchronous rather than slow wall movement with greatly increased spread in the timing of peak outward velocity in the three regions. It was also apparent that this asynchrony was not random. Outward movement of the anterior region consistently preceded that of the inferior, and frequently started before mitral valve opening. This regional difference in timing was apparent throughout diastole until the end of rapid outward movement. In six patients, another very characteristic pattern of wall motion was seen- after initial outward motion, one region moved inwards again during ventricular filling, at the same time as another region moved outwards. This resulted in an oscillation of the whole ventricular cavity, at a frequency of 4 to $6 \mathrm{~Hz}$.

These results exclude the simple hypothesis that rate of filling is the only determinant of diastolic wall motion in mitral stenosis. It is possible that regional motion was modified by the presence of thickened or shortened papillary muscles. In order to investigate this possibility three patients with obstructed StarrEdwards mitral prostheses in whom the mitral subvalvular apparatus had been removed were studied. The results showed patterns of wall motion identical to those seen in patients with naturally occurring mitral stenosis (unpublished observation). Another possibility is that this abnormal wall motion was related in some way to the left ventricular posterobasal hypokinesia reported to occur in patients with mitral stenosis by Heller and Carleton. ${ }^{9}$ There was no statistical difference, however, in the overall amplitude of systolic inward wall motion for the three regions in our patients. If regional hypokinesis was not present during systole, it could not have affected wall motion during diastole.

Additional factors must be invoked to explain the diastolic pattern of regional left ventricular wall motion in mitral stenosis. Its nature is not dependent on mechanical obstruction to inflow or on structural abnormalities associated with the disease itself. We have previously suggested ${ }^{10}$ that a major determinant of normal early diastolic phase of rapid filling is rapid thinning of the myocardium, with resulting outward motion of endocardium. The extent to which this process occurs varies with position in the cavity, being maximum in the mid-portion of the free wall. ${ }^{11} \mathrm{It}$ is independent of filling ${ }^{10}$ and so is probably energised by elastic forces generated during the previous systole. ${ }^{12}$ In patients with ischaemic heart disease, asynchronous outward wall motion during isovolumic relaxation disturbs this normal filling mechanism, and rapid wall thinning may prematurely occur before mitral valve opening. Again this process is virtually confined to the mid-portion of the anterior free wall, regardless of the distribution of the coronary artery disease itself. ${ }^{13}$ The present results allow these observations to be extended. Instead of filling rate being zero as in isovolumic relaxation, it is low and fixed for a significant portion of diastole in patients with mitral stenosis, which again allows the possibility of mismatch between regional wall motion and filling to be explored, but over a much longer period. As in ischaemic heart disease during isovolumic relaxation, so in mitral stenosis after mitral valve opening, the filling rate is not matched to the tendency of the ventricle to regain its diastolic configuration, and asynchronous wall motion occurs. Once more, this ten- 
dency is most striking along the anterior wall whose motion precedes that of the apex and the inferior wall. Indeed, it appears that on occasion the magnitude of these forces may be so great as to induce oscillation of the fluid within it, when early outward motion of the anterior wall is followed by inward motion during filling, with compensatory motion elsewhere in the ventricle, usually along the inferior wall. Such oscillations have previously been invoked to explain certain diastolic events in patients with ischaemic heart disease, ${ }^{14}$ and the present observations provide direct evidence for their existence. Since the resonant frequency of any body of fluid is determined in part by its mass, the relatively low rate of increase in diastolic left ventricular volume allows a longer time for their development than in the normal, when volume changes rapidly.

The present results are thus compatible with previous observations on diastolic wall motion in man and provide further evidence that regional factors within the ventricle itself, probably related to fibre architecture, must be invoked to explain normal and abnormal filling patterns. They also illustrate that regional abnormalities of wall motion need not necesarily represent the presence of regional disease, but may merely result from the unmasking of physiological mechanisms operating in abnormal conditions. Such observations on patients with mitral stenosis may thus shed further light on these diastolic events, underlining the highly organised and complex nature of the normal processes of left ventricular filling in man.

\section{References}

1 Gorlin R, Gorlin SG. Hydraulic formula for calculation of the area of stenotic mitral valve, other cardiac valves, and central circulatory shunts. Am Heart $\mathcal{F}$ 1951; 41: $1-29$.

2 Gibson D, Brown D. Measurement of instantaneous left ventricular dimension and filling rate in man, using echocardiography. Br Heart f 1973; 35: 1141-9.
3 Kalmanson D, Veyrat C, Bouchareine F, Degroote A. Non-invasive recording of mitral valve flow velocity patterns using pulsed Doppler echocardiography. Br Heart f 1977; 39: 517-28.

4 Thuillez C, Theroux P, Bourassa MG, et al. Pulsed Doppler echocardiographic study of mitral stenosis. Circulation 1980; 61: 381-7.

5 Hammermeister KE, Warbasse JR. The rate of change of left ventricular volume in man. II. Diastolic events in health and disease. Circulation 1974; 49: 739-47.

6 Gibson DG, Brown DJ. Continuous assessment of left ventricular shape in man. Br Heart $\mathcal{F}$ 1975; 37: 904-10.

7 Gibson DG, Prewitt TA, Brown DJ. Analysis of left ventricular wall movement during isovolumic relaxation and its relation to coronary artery disease. $B r$ Heart $\mathcal{F} 1976$; 38: 1010-9.

8 Wang RYC, Chen WC, Lee PK, Shen E. Coronary arteriographic findings among Chinese patients in Hong Kong. Chin Med f 1982; 21: 285-7.

9 Heller SJ, Carleton RA. Abnormal left ventricular contraction in patients with mitral stenosis. Circulation 1970; 42: 1099-110.

10 Gibson DG, Traill TA, Brown DJ. Changes in left ventricular free wall thickness in patients with ischaemic heart disease. Br Heart $\mathcal{F}$ 1977; 39: 1312-8.

11 Shapiro E, Marier DL, St. John Sutton MG, Gibson DG. Regional non-uniformity of wall dynamics in normal left ventricle. Br Heart $\mathcal{F}$ 1981; 45: 264-70.

12 Gibson DG, Greenbaum R, Marier DL, Brown DJ. Clinical significance of early diastolic changes in left ventricular wall thickness. Eur Heart Y' 1980 ; 1 (suppl A): 157-63.

13 Greenbaum RA, Gibson DG. Regional non-uniformity of left ventricular movement in man. Br Heart $\mathcal{F}$ 1981; 45: 29-34.

14 Matheos M, Shapiro E, Oldershaw PJ, Sacchetti R, Gibson DG. Non-invasive assessment of changes in left ventricular relaxation by combined phono-, echo-, and mechanocardiography. Br Heart f 1982; 47: 253-60.

Requests for reprints to Dr D G Gibson, Cardiac Department, Brompton Hospital, Fulham Road, London SW3 6HP. 